\title{
Does Ketamine Have Anti-Suicidal Properties? Current Status and Future Directions
}

\author{
Rebecca B. Price $\cdot$ Sanjay J. Mathew
}

Published online: 26 February 2015

(C) Springer International Publishing Switzerland 2015

\begin{abstract}
Ketamine, a widely used anesthetic agent, is currently being investigated as a novel therapeutic for depression and suicidality. Ketamine has garnered substantial attention from researchers, clinicians, media outlets, and patients alike, but numerous questions remain. One of the compelling features of ketamine is the rapidity of its antidepressant effects, which peak just $24 \mathrm{~h}$ after infusion, setting it apart from other existing treatments. Ketamine's rapid time course has inspired research efforts to explore its potential as a life-saving therapy for patients at imminent risk of suicide. In this article, we review current evidence supporting the rapid effects of ketamine on suicidal ideation in the context of unipolar and bipolar depression. We then discuss several future directions that are necessary before ketamine can be considered a viable treatment option for suicidality in clinical settings. These include: testing for a specific anti-suicidal effect-separate from overall antidepressant effects-to ascertain whether ketamine might hold promise for a broader class of suicidal patients; ensuring that acute benefits of ketamine can be prolonged over a clinically meaningful timeframe; and developing a better understanding of the mechanisms by which ketamine might reduce suicide risk. Such efforts will enable the field to more accurately assess the potential of
\end{abstract}

\footnotetext{
R. B. Price $(\square)$

Department of Psychiatry, University of Pittsburgh School of Medicine, 3811 O'Hara St., Pittsburgh, PA 15213, USA

e-mail: rebecca.price@stanfordalumni.org

S. J. Mathew

Michael E. DeBakey VA Medical Center, Houston, TX, USA

S. J. Mathew

Menninger Department of Psychiatry and Behavioral Sciences, Baylor College of Medicine, Houston, TX, USA
}

ketamine, as well as its limitations, allowing for appropriate placement within the context of comprehensive clinical care for suicide prevention.

\section{Key Points}

Ketamine has shown initial promise as a rapid reducer of suicidal ideation in treatment-resistant unipolar and bipolar depression.

More research is needed to (a) explore whether ketamine has suicide-specific effects that generalize beyond depressed patients with low to moderate suicide risk; (b) test whether acute benefits can be prolonged; and (c) understand the mechanisms of ketamine's anti-suicidal properties.

\section{Introduction}

Ketamine is a non-competitive $N$-methyl-D-aspartate (NMDA) glutamate receptor antagonist that has shown promise as a novel therapeutic for unipolar and bipolar depression [1,2]. The rapidity of ketamine's antidepressant effects, together with its efficacy in patients failing to respond to conventional antidepressant treatment (e.g., [3, 4]), has sparked interest in its potential as an anti-suicide treatment. Depressive symptom reductions are typically observed within $2 \mathrm{~h}$ of ketamine infusion, and peak by $24 \mathrm{~h}$ post-infusion, making ketamine a theoretically attractive candidate for securing the safety of patients who are at imminent risk of death by suicide. Given that suicide is a leading cause of death and disease burden in the US and 
worldwide, with approximately one million annual deaths globally, the prospect of saving lives via rapid reduction in suicidality (a term which we use to encompass both thoughts of suicide [suicidal ideation; SI] and overt suicidal behaviors) has substantial public health ramifications [5].

Suicide rates have continued to rise in recent decades, despite ongoing clinical and research efforts [5, 6]. Existing treatment options for those at imminent suicide risk include conventional antidepressants, electroconvulsive therapy (ECT), psychotherapy (e.g., cognitive therapy for suicidal patients; dialectical behavior therapy), lithium, and clozapine. Although each of these options has some degree of established efficacy for reducing markers of suicide risk [7], only clozapine has a US Food and Drug Administration (FDA) approved indication for suicidal behavior (in the context of psychotic disorders only), and a controversial FDA black box warning alerts prescribers to the possibility of increased suicidality in depressed youth treated with selective serotonin reuptake inhibitors, further complicating treatment decisions. Furthermore, a substantial portion of patients (e.g., 50-70\%) will prove resistant to any one existing approach [8], and algorithms for efficient patienttreatment matching have proven elusive.

An additional limitation of each of these optionscompounded by the problem of treatment resistance-is the relative sluggishness of therapeutic onset, leaving a window of danger during which suicidal acts may be pursued. For instance, it is well established that conventional antidepressants take weeks to improve depression symptoms including SI. Alternatives such as ECT are considered more rapid, but in a sample of patients at high acute suicide risk, six ECT sessions (conducted over a 2-week period) were needed before clinician-rated suicidality was eradicated in at least half of patients [9]. Finally, while treatments for acute suicidality are frequently offered in the context of inpatient hospitalization to promote immediate patient safety, hospitalization is costly to the healthcare system and viewed as largely undesirable by both patients and clinicians. Furthermore, death by suicide occurs even within the inpatient context [10] and is particularly likely in the period immediately post-discharge [11]. A safe, feasible, cost-effective, and less stigmatizing means to efficiently reduce suicide risk in clinical settings would therefore be highly desirable.

In light of these limitations in the clinical management of acute suicidality, ketamine's potential to rapidly impact suicidality has been explored in several studies. However, numerous questions remain to be answered before this treatment can be considered a reasonable option in clinical settings. In this Current Opinion article, we review published studies on the anti-suicidal properties of ketamine. We then discuss several future directions for research, including studies necessary to (a) establish a specific anti- suicidal effect of ketamine, (b) ensure that acute effects of ketamine can be extended, and (c) understand the mechanisms via which reductions in suicidality are achieved. Additional practical considerations, including dose optimization, infusion speed, and appropriate credentialing of medical professionals administering ketamine, are beyond the scope of the current review, but will be key factors to address prior to clinical adoption.

\section{Studies of Ketamine's Anti-Suicidal Effects}

\subsection{Uncontrolled Studies}

The first published report examining ketamine's effects on suicidality was an open-label trial of intravenous ketamine in a sample of 26 patients with treatment-resistant major depression (TRD) [12]. Twenty-four hours after a single ketamine infusion $(0.5 \mathrm{mg} / \mathrm{kg}$ over $40 \mathrm{~min})$, large reductions (Cohen's $d=1.4$ ) in SI were observed on the clinician-rated suicide item of the Montgomery-Asberg Depression Rating Scale (MADRS; [13]), with $81 \%$ of patients rating 0 or 1 on the MADRS 6-point scale. To test whether these rapid effects could be sustained through repeated ketamine infusions, a subset of the patients $(n=10)$ were recruited into a repeated-dose open-label trial. Nine out of ten patients were classified as responders to a first infusion (based on a $50 \%$ reduction in overall MADRS score) and then received five additional infusions given thrice-weekly over the course of 2 weeks. In all nine patients, MADRS suicide item scores were reduced to 0 and remained at 0 or 1 throughout the 2 -week trial.

Similar results were obtained in a sample of 33 TRD patients following a single infusion [14] at the same dose as in the Price et al. report above. Significant reductions on the Scale for Suicide Ideation (SSI), as well as the suicide items from several depression inventories, were documented as early as 40 min after the start of the infusion (i.e., immediately after the infusion concluded), with gains maintained up to $230 \mathrm{~min}$ post-infusion (no data presented for later time points). Among patients who scored above a clinically significant cut-off point on the SSI at baseline $(\geq 4)$, all fell below this cut-off point by 80 min post-infusion, and $60 \%$ reached an SSI score of 0 within $80 \mathrm{~min}$. These rapid and significant reductions at 40 and 230 min post-infusion were later replicated in 27 TRD patients receiving open-label ketamine (at the same dose) in India [15]; however, contrary to all other studies reporting on 24-h outcomes, SI scores returned to baseline levels by $24 \mathrm{~h}$ in this sample. Finally, a sample of ten TRD patients were given serial doses of ketamine $(0.5 \mathrm{mg} / \mathrm{kg}$ over $100 \mathrm{~min}$ ) twice weekly until either remission of general depression symptoms was obtained or the 
maximum of four doses was reached [16]. From baseline to $24 \mathrm{~h}$ after final infusion, SI scores were significantly reduced on both self-report and clinician-rated measures.

The anti-suicide effects of ketamine have also been studied in routine clinical contexts. In a sample of 14 unipolar depression patients presenting to the psychiatric ER with SI, an intravenous bolus of ketamine $0.20 \mathrm{mg} / \mathrm{kg}$ delivered over 1-2 min (actual doses based on repeat weight assessment ranged from 0.16 to $0.26 \mathrm{mg} / \mathrm{kg}$ ) significantly reduced scores on the MADRS suicide item beginning 40 min post-infusion, with gains maintained for 10 consecutive days post-infusion during ongoing, conventional treatment [17]. The efficacy of this bolus infusion suggests that additional studies are warranted to assess whether slow infusions of $0.5 \mathrm{mg} / \mathrm{kg}$, used in the vast majority of studies to date, are in fact necessary and optimal. In an ECT clinic, 28 TRD patients received either three doses $(n=15)$ or six doses $(n=13)$ of ketamine $(0.5 \mathrm{mg} / \mathrm{kg}$ over $40 \mathrm{~min})$ over 3 weeks, while continuing other psychotropic medications [18]. Scores on the suicide item of the Hamilton Rating Scale for Depression (HAMD) were significantly reduced within $6 \mathrm{~h}$ of the initial infusion and, in those individuals who obtained a $50 \%$ decrease in total depression symptoms $(n=8)$, improvements in SI were sustained until the final assessment 4-7 days after the last infusion. Finally, two case reports have found marked beneficial effects of oral [19] and intravenous [20] ketamine when given to patients with intractable SI in clinical contexts.

\subsection{Randomized Controlled Trials}

A randomized controlled trial (RCT) examined the antisuicidal properties of ketamine in 15 patients with bipolar depression (BD) receiving ketamine $(0.5 \mathrm{mg} / \mathrm{kg}$ over $40 \mathrm{~min}$ ) or saline infusion in a double-blind, cross-over design [21]. Ketamine, but not saline, reduced scores on the MADRS suicide item beginning at $40 \mathrm{~min}$ post-infusion and continuing through the 72-h assessment (with a large between-group effect size of $d=2.09$ across all time points). SI levels no longer differed across arms when reassessed at Day 7.

In a second RCT, ketamine was compared with midazolam, a psychoactive agent selected to mimic the sedative and anesthetic properties of ketamine [22]. Fifty-seven TRD patients were randomized to ketamine or midazolam in a 2:1 ratio. Twenty-four hours post-infusion, ketamine, but not midazolam, reduced scores on a composite index comprising three measures (the Beck Suicide Scale and the suicide items of the MADRS and the Quick Inventory of Depressive Symptoms) with a large between-groups effect $(d=0.82)$. Ketamine was associated with total eradication of SI across all three measures in $54 \%$ of the sample at
$24 \mathrm{~h}$, compared with $24 \%$ of the midazolam group. As in this research group's previous open-label study, ketamine also reduced implicit suicide-related cognition [Implicit Association Test (IAT) 'Escape $=\mathrm{Me}$ ' associations]; however, this implicit effect could not be definitively shown to be larger in the ketamine than the midazolam group.

In summary, results to date suggest a subanesthetic dose of ketamine shows initial promise in rapidly reducing SI (Table 1), but numerous questions remain for future research.

\section{Future Directions}

\subsection{Establishing the Specificity and Generalizability of Anti-Suicidal Properties}

Suicide risk is elevated in a wide range of diagnostic groups [23] and exhibits unique genetic variance that can be inherited separately from mood disorder risk [24]. It therefore may be most accurately viewed as a transdiagnostic dimension on which individuals with and without primary mood disorders vary. Although ketamine has shown initial promise in the treatment of suicidal ideation, conclusions from the extant literature are limited by the fact that suicidality has been examined exclusively in the context of (unipolar or bipolar) depression treatment trials which have generally excluded individuals at imminent risk for suicide. As a result, it remains unclear whether ketamine has suicide-specific properties that constitute more than simply a by-product or corollary of depression reduction. Studies to date have produced conflicting findings on this issue. In two studies of TRD patients by Price et al. [12, 22], SI reductions $24 \mathrm{~h}$ post-infusion were statistically mediated by depression reductions (measured both at 24 and $4 \mathrm{~h}$ post-infusion). In the context of these relatively small, chronically depressed samples, ketamine could therefore not be shown to have a unique effect on SI over and above depression alleviation, but produced SI reductions in concert with overall depression improvement.

By contrast, Ballard et al. [25] pooled patient-level data across four previously published trials of ketamine in TRD and BD to examine the relationship between SI reduction and broader improvements in anxiety and depression. In a subset of patients $(n=57)$ who had significant SI at baseline and participated in placebo-controlled RCTs comparing ketamine and saline, ketamine infusion was related to decreased SI (assessed by the HAMD suicide item) and lesser wish to die and greater wish to live (assessed by relevant individual items on the SSI) from $40 \mathrm{~min}$ to 3 days post-infusion, even after controlling for depression and anxiety symptoms at each time point. 


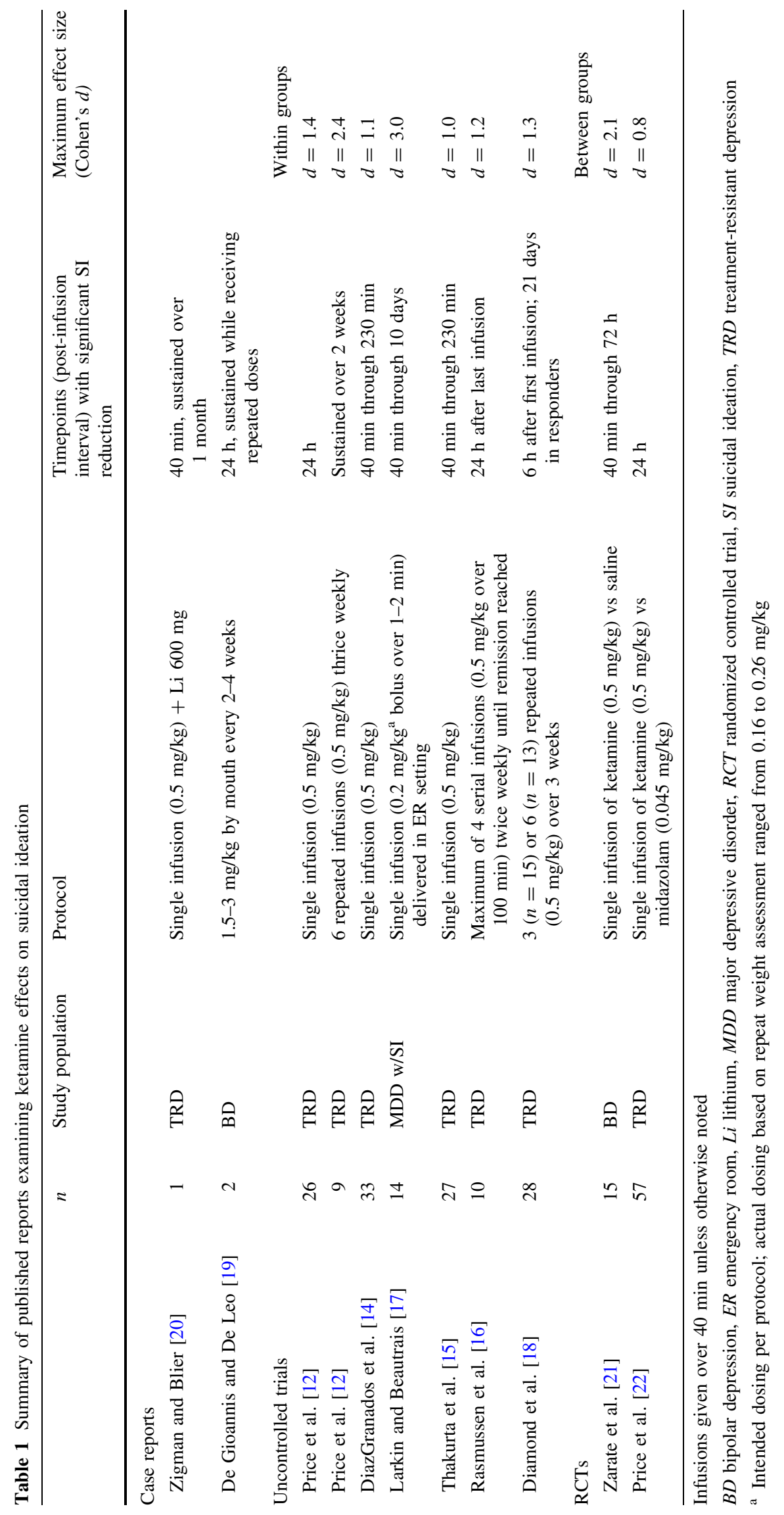


Although formal statistical mediation analysis was not reported, the persistent association between ketamine and SI indices suggests reductions in anxiety and depression did not fully account for the anti-SI effects of ketamine in these patients. The differing results may stem from the inclusion of $\mathrm{BD}$ in addition to TRD patients, a relatively large number of patients with significant SI at baseline, and/or multiple post-infusion timepoints, all of which may have allowed for a greater degree of depression-independent variance in SI to emerge.

It is important to note that studies to date have used measures of SI as indicators of decreased suicide risk following ketamine infusion. However, current SI is a relatively imprecise proxy for overt suicidal behaviors like suicide completion [26]. Inclusion of additional proxy measures as outcomes (e.g., psychic anxiety, anhedonia, impulsivity [27]) could help to extend SI findings. Suicidal behaviors themselves are comparatively rare and therefore more difficult to quantify in clinical trials research, particularly given that ketamine trials to date have often excluded patients deemed at imminent risk of suicide. Specifically recruiting high-risk samples (e.g., suicidal inpatients/ER visitors; suicide attempters) should increase statistical power to examine whether ketamine prevents suicidal behavior in addition to reducing ideation, although large sample sizes would still be required. Furthermore, recruiting a sample defined on the basis of suicide risk, as opposed to mood disorder diagnosis, affords the opportunity to ascertain whether the effects of ketamine on suicidal indices might generalize to a wider range of patients, including those not meeting formal criteria for a mood disorder. Further research is clearly required to assess this possibility, and caution is warranted in patients who may be at increased risk due to the abuse potential of ketamine. Several such trials in high-risk samples are recently concluded (ClinicalTrials.gov identifier: NCT01507181) or ongoing (NCT01892995; NCT01887990) and results should be forthcoming.

\subsection{Prolonging Relief from Suicidality}

As reviewed above, in a single uncontrolled study, repeated ketamine infusions have been shown to continuously eradicate SI over a period of 2 weeks [12]. However, the longterm effects of repeated ketamine infusions, both desirable (e.g., protracted protection from suicide) and undesirable (e.g., neurocognitive impairments, increasing abuse liability), remain inadequately studied and poorly understood. A critical, unanswered question is whether ketamine might eventually find a role within a larger, comprehensive treatment strategy designed to effect change in a lasting way, without necessitating repeated infusions in perpetuity. Anecdotal reports suggest the possibility for prolonged improvements in depression and SI lasting 1-3 months after ketamine treatment [20, 28, 29], consistent with enduring antidepressant-like effects observed in rats following repeated administrations [30]. However, such responses are not normative in trials to date, and without a proven strategy to reliably extend the short-term benefits of ketamine, there is a serious risk of rebound in suicidality that could prove dangerous without appropriate monitoring.

An improved understanding of the mechanisms of the anti-suicidal effects of ketamine would facilitate efforts to extend ketamine's acute effects, either through development of alternative, less invasive treatments that target the same mechanisms, or through synergistic treatment combinations. For instance, the hypothesis that response to an initial infusion with ketamine could be prolonged by prescribing a similar glutamatergic agent in pill form (riluzole) was tested in TRD samples; however, riluzole showed no efficacy in these trials [31, 32]. Alternative synergistic combinations are certainly possible using a wide range of existing treatments that may be more durable, but slower to act (e.g., cognitive behavioral therapies, conventional antidepressants, ECT), and/or through novel therapeutics targeting key mechanisms. Several candidate mechanisms, which could help foster such clinical trial hypotheses, are discussed below.

\subsection{Delineating the Mechanisms of the Effects of Ketamine on Suicidality}

\subsubsection{Neuroinflammatory Pathways}

Neurobiological mechanisms of the antidepressant effects of ketamine are the focus of substantial ongoing research efforts. Hypothesized mechanisms have been reviewed elsewhere [33], and include alterations in glutamatergic and dopaminergic function and neuroplasticity (e.g., brainderived neurotrophic factor). As noted above, the effects of ketamine on suicidality may be part and parcel of its overall antidepressant benefit, meaning that the neurobiological mechanisms may be highly overlapping. Methodologies including genetics, neuroimaging, and sleep architecture studies will likely continue to inform our understanding of both antidepressant and anti-suicide mechanisms [34].

One particularly noteworthy line of research points to a neuroinflammatory pathway that may be both uniquely altered in suicidal patients and relevant to ketamine's pharmacology, suggesting a potential suicide-specific mechanism of action. The amino acid tryptophan is a precursor for numerous neurochemicals, with the majority of tryptophan being metabolized to kynurenine, particularly during states of inflammation [35]. Kynurenine is, in turn, metabolized through two separate pathways to 
become either quinolinic acid, an NMDA receptor agonist, or kynurenic acid, an NMDA receptor antagonist. In a sample of recent suicide attempters, the total amount of quinolinic acid in the cerebrospinal fluid, as well as the ratio of quinolinic to kynurenic acid, was found to be elevated in comparison with healthy controls, suggesting a tip in the balance towards NMDA receptor stimulation [36]. Quinolinic acid was particularly elevated in participants with high scores on the Suicide Intent Scale and those who had attempted using violent means. A subset of participants returned for reassessment approximately 6 months after the attempt and showed decreased quinolinic acid levels that were comparable to healthy controls.

Together with a larger literature suggesting elevated pro-inflammatory and inflammatory cytokines in individuals at risk of suicide [37], these abnormalities within the kynurenine pathway suggest a state of immune activation and NMDA receptor stimulation that is posited to confer suicide risk. One compelling hypothesis is that ketamine, an NMDA receptor antagonist, may help to remedy this imbalance by decreasing NMDA throughput, resulting in a less suicide-prone state. This hypothesis has yet to be tested in suicidal patients receiving ketamine.

\subsubsection{Cognitive and Behavioral Mechanisms}

In addition to its numerous neurobiological effects, ketamine also exerts a dramatic influence on patients at the level of conscious experience, thought, and behavior by rapidly relieving depressive symptoms that may have proven intractable for decades (e.g., average duration of current episode $>12$ years; $[4,31])$. It is possible that even a transient experience of this sort could affect a patient's orientation and level of engagement with treatment in a manner that is protective against suicide, as discussed below. However, given that depression is a placebo-responsive illness [38], it is also likely that patient's expectancies amplify any genuine therapeutic effect of ketamine, particularly given that the majority of studies to date have been uncontrolled. Additional placebo-controlled trials with adequate blinding, at both the patient and clinician level, are necessary to test whether the effects of ketamine are indeed superior to placebo, both statistically and clinically speaking.

Hopelessness is a key factor in cognitive models of suicide and one of the few variables that reliably and prospectively predicts suicidal behaviors themselves [26, 39]. Hopelessness was found to be acutely reduced by ketamine in two TRD studies [14, 22], which could reflect that hope is naturally instilled by experiencing long-sought therapeutic benefit. In an RCT comparing ketamine and midazolam [22], there was a trend towards greater reduction in hopelessness following ketamine compared with midazolam, and hopelessness decreases were strongly related to decreased SI. Although these results are suggestive, full statistical mediation requirements were not satisfied, which could reflect insufficient power due to the study design (i.e., recruitment of a TRD sample with heterogeneous levels of SI).

This study also found a trend towards statistical mediation of SI improvements by euphoria, which was assessed at the conclusion of the infusion when the transient mood-lifting effects of ketamine were still evident. Ketamine-treated patients who reported the largest acute increases in euphoria were more likely to report substantial SI decreases at the 24-h assessment (long after euphoria had subsided). This pattern could reflect either increased efficacy of glutamatergic target engagement during the infusion-resulting in both a larger acute 'high' and greater therapeutic benefit - and/or a downstream cognitive effect whereby a transient euphoric experience helps to alleviate the desire to die up to $24 \mathrm{~h}$ later. By contrast, dissociative symptoms during the infusion, another side effect of ketamine's glutamatergic mechanisms, were not significantly related to SI decreases. Notably, dissociative symptoms have been reported to correlate with the antidepressant effects of ketamine [40], suggesting a possible dichotomy between antidepressant and anti-suicide mechanisms. These findings highlight the need to further explore the role of the acute euphoric, dissociative, and other cognitive (e.g., mystical; [41]) effects of ketamine in producing both antidepressant and anti-suicidal effects, particularly given that other agents with transient mood-elevating properties (e.g., stimulants, opiates) have had limited clinical impact and are generally proscribed due to abuse liability [42].

Anhedonia is a behavioral dimension of depression that has been previously linked to proximal risk of suicide completion [27]. In a recent report, anhedonia levels in a sample of $36 \mathrm{BD}$ patients were reduced for up to 14 days following a single infusion of ketamine (but not saline), even after controlling for total depression at each timepoint [43]. This pattern suggests a specific benefit of ketamine on anhedonia, over and above its effect on other depression symptoms, which could potentially contribute to decreased immediate suicide risk, particularly if a state of decreased anhedonia allows for greater engagement with protective factors in the environment (e.g., increased social engagement). Decreased anhedonia was further linked to a pattern of increased glucose metabolism in reward-processing regions of the brain (dorsal anterior cingulate; putamen). The role of these motivation-related mechanisms in the antisuicidal effects of ketamine clearly warrants further study.

Finally, implicit suicide-related cognition, assessed behaviorally using a performance-based measure, was reduced following ketamine infusion in two studies [12, 22]. Specifically, associations between escape- and self-related words on the IAT were reduced, with the degree of change 
on IAT performance tracking changes in explicit SI. Implicit suicide-related cognition, assessed by the IAT, is also a strong prospective predictor of future suicide attempts, outperforming a variety of demographic and clinical indicators [44]. Reductions in implicit associations could therefore represent a hypothetical pathway to protracted protection against suicide following ketamine, particularly if such changes can be shown to be stable over a clinically meaningful period of time. A similar model has recently been proposed for the serotonergic antidepressants, wherein acute drug effects on performance-based measures of cognitive-affective processing are posited to explain downstream therapeutic benefits [45]. Additional studies incorporating long-term follow-up and repeated IAT assessment would be needed to explore this possibility.

\section{Conclusions}

Studies to date suggest intravenous ketamine produces substantial reductions in SI in patients with unipolar and bipolar depression, in both uncontrolled and randomized controlled trials (Table 1). Large reductions have been observed as early as 40 min post-infusion, with improvements maintained for variable amounts of time ranging from $230 \mathrm{~min}$ up to 10 days post-infusion (with ongoing conventional care). However, research published to date leaves numerous critical and clinically relevant questions unanswered, suggesting clinical adoption is not yet warranted. Further studies are indicated to explore the specificity, generalizability, durability, and mechanisms of the anti-suicidal effects of ketamine. The promise of this line of work is that suicidal patients in need of life-saving measures will one day have access to rapid, durable, safe, and effective relief.

\begin{abstract}
Acknowledgments Dr. Price is supported by a Career Development Award from NIMH (K23MH100259). Dr. Mathew's work was supported in part by National Institute of Mental Health (NIMH) grant RO1MH081870. Dr. Mathew is supported with resources and the use of facilities at the Michael E. DeBakey VA Medical Center, Houston, TX, USA, and by the Marjorie Bintliff Johnson and Raleigh White Johnson, Jr. Chair for Research in Psychiatry at Baylor College of Medicine. In the past 12 months, Dr. Mathew has received consulting fees from Cerecor, Genentech, Naurex, and research support from AstraZeneca, Janssen Research and Development, and Otsuka. He has received medication from Sanofi for a NIH-funded clinical trial.

We gratefully acknowledge Dr. David Brent for providing comments on a previous version of this manuscript. Dr. Price reports no conflicts of interest.
\end{abstract}

\section{References}

1. Aan Het Rot M, Zarate CA, Charney DS, Mathew SJ. Ketamine for depression: where do we go from here? Biol Psychiatry. 2012;72:537-47. doi:10.1016/j.biopsych.2012.05.003.
2. Mathew SJ, Shah A, Lapidus K, Clark C, Jarun N, Ostermeyer B, et al. Ketamine for treatment-resistant unipolar depression: current evidence. CNS Drugs. 2012;26(3):189-204. doi:10.2165/ 11599770-000000000-00000.

3. Zarate CA, Singh JB, Carlson PJ, Brutsche NE, Ameli R, Luckenbaugh DA, et al. A randomized trial of an $N$-methyl-Daspartate antagonist in treatment-resistant major depression. Arch Gen Psychiatry. 2006;63:856-64. doi:10.1001/archpsyc.63.8.856.

4. Murrough JW, Iosifescu DV, Chang LC, Al Jurdi RK, Green CM, Perez AM, et al. Antidepressant efficacy of ketamine in treatment-resistant major depression: a two-site randomized controlled trial. Am J Psychiatry. 2013;170(10):1134-42. doi:10. 1176/appi.ajp.2013.13030392.

5. Nock MK, Borges G, Bromet EJ, Cha CB, Kessler RC, Lee S. Suicide and suicidal behavior. Epidemiol Rev. 2008;30:133-54. doi:10.1093/epirev/mxn002.

6. Kessler RC, Berglund P, Borges G, Nock M, Wang PS. Trends in suicide ideation, plans, gestures, and attempts in the United States, 1990-1992 to 2001-2003. JAMA. 2005;293(20):2487-95. doi:10.1001/jama.293.20.2487.

7. Al Jurdi RK, Swann A, Mathew SJ. Psychopharmacological agents and suicide risk reduction: ketamine and other approaches. 2015. (in press).

8. Rush J, Trivedi MH, Wisniewski SR, Nierenberg A, Stewart JW, Warden D, et al. Acute and longer-term outcomes in depressed outpatients requiring one or several treatment steps: a STAR*D report. Am J Psychiatry. 2006;163:1905-17. doi:10.1176/appi. ajp.163.11.1905.

9. Fink M, Kellner $\mathrm{CH}, \mathrm{McC}$ all WV. The role of ECT in suicide prevention. J ECT. 2014;30(1):5-9. doi:10.1097/YCT. 0b013e3182a6ad0d.

10. Watts BV, Young-Xu Y, Mills PD, DeRosier JM, Kemp J, Shiner B, et al. Examination of the effectiveness of the mental health environment of care checklist in reducing suicide on inpatient mental health units. 2012;69:588-92. doi:10.1001/archgenpsychiatry.2011. 1514.

11. Qin P, Nordentoft M. Suicide risk in relation to psychiatric hospitalization: evidence based on longitudinal registers. Arch Gen Psychiatry. 2005;62(4):427-32. doi:10.1001/archpsyc.62.4. 427.

12. Price RB, Nock MK, Charney DS, Mathew SJ. Effects of intravenous ketamine on explicit and implicit measures of suicidality in treatment-resistant depression. Biol Psychiatry. 2009;66(5):522-6. doi:10.1016/j.biopsych.2009.04.029.

13. Montgomery SA, Asberg M. A new depression scale designed to be sensitive to change. Br J Psychiatry. 1979;134:382-9.

14. DiazGranados N, Ibrahim L, Brutsche NE, Ameli R, Henter ID, Luckenbaugh DA, et al. Rapid resolution of suicidal ideation after a single infusion of an NMDA antagonist in patients with treatment-resistant major depressive disorder. J Clin Psychiatry. 2010;71:1605-11. doi:10.4088/JCP.09m05327blu.Rapid.

15. Thakurta RG, Das R, Bhattacharya AK, Saha D, Sen S, Singh OP, et al. Rapid response with ketamine on suicidal cognition in resistant depression. Indian J Psychol Med. 2012;34(2):170-5. doi:10.4103/0253-7176.101793.

16. Rasmussen KG, Lineberry TW, Galardy CW, Kung S, Lapid MI, Ba Palmer, et al. Serial infusions of low-dose ketamine for major depression. J Psychopharmacol. 2013;27:444-50. doi:10.1177/ 0269881113478283.

17. Larkin GL, Beautrais AL. A preliminary naturalistic study of low-dose ketamine for depression and suicide ideation in the emergency department. Int J Neuropsychopharmacol. 2011;14:1127-31. doi:10.1017/S1461145711000629.

18. Diamond PR, Farmery AD, Atkinson S, Haldar J, Williams N, Cowen PJ, et al. Ketamine infusions for treatment resistant depression: a series of 28 patients treated weekly or twice weekly in 
an ECT clinic. J Psychopharmacol. 2014;28(6):536-44. doi:10. 1177/0269881114527361.

19. De Gioannis A, De Leo D. Oral ketamine augmentation for chronic suicidality in treatment-resistant depression. Aust N Z J Psychiatry. 2014;48(7):686. doi:10.1177/0004867414520754.

20. Zigman D, Blier P. Urgent ketamine infusion rapidly eliminated suicidal ideation for a patient with major depressive disorder: a case report. J Clin Psychopharmacol. 2013;33(2):270-2. doi:10. 1097/JCP.0b013e3182856865.

21. Ca Zarate, Brutsche NE, Ibrahim L, Franco-Chaves J, Diazgranados N, Cravchik A, et al. Replication of ketamine's antidepressant efficacy in bipolar depression: a randomized controlled add-on trial. Biol Psychiatry. 2012;71:939-46. doi:10. 1016/j.biopsych.2011.12.010.

22. Price RB, Iosifescu DV, Murrough JW, Chang LC, Al Jurdi RK, Iqbal SZ, et al. Effects of ketamine on explicit and implicit suicidal cognition: a randomized controlled trial in treatment-resistant depression. Depress Anxiety. 2014;31(4):335-43. doi:10. 1002/da.22253.

23. Nock MK, Kessler RC. Prevalence of and risk factors for suicide attempts versus suicide gestures: analysis of the National Comorbidity Survey. J Abnorm Psychol. 2006;115(3):616-23. doi:10.1037/0021-843x.115.3.616.

24. Brent DA, Melhem N. Familial transmission of suicidal behavior. Psychiatr Clin N Am. 2008;31(2):157-77. doi:10.1016/j.psc. 2008.02.001.

25. Ballard ED, Ionescu DF, Vande Voort JL, Niciu MJ, Richards EM, Luckenbaugh DA, et al. Improvement in suicidal ideation after ketamine infusion: relationship to reductions in depression and anxiety. J Psychiatr Res. 2014;58:161-6. doi:10.1016/j. jpsychires.2014.07.027.

26. Beck AT, Brown GK, Steer RA, Dahlsgaard KK, Grisham JR. Suicide ideation at its worst point: a predictor of eventual suicide in psychiatric outpatients. Suicide Life Threat Behav. 1999;29(1):1-9.

27. Fawcett J, Scheftner WA, Fogg L, Clark DC, Young MA, Hedeker D, et al. Time-related predictors of suicide in major affective disorder. Am J Psychiatry. 1990;147(9):1189-94.

28. Aan Het Rot M, Collins KA, Murrough JW, Perez AM, Reich DL, Charney DS, et al. Safety and efficacy of repeated-dose intravenous ketamine for treatment-resistant depression. Biol Psychiatry. 2010;67(2):139-45.

29. Murrough JW, Perez AM, Pillemer S, Stern J, Parides MK, Aan Het Rot M, et al. Rapid and longer-term antidepressant effects of repeated ketamine infusions in treatment-resistant major depression. Biol Psychiatry. 2012;. doi:10.1016/j.biopsych.2012.06.022.

30. Parise EM, Alcantara LF, Warren BL, Wright KN, Hadad R, Sial $\mathrm{OK}$, et al. Repeated ketamine exposure induces an enduring resilient phenotype in adolescent and adult rats. Biol Psychiatry. 2013;74:750-9. doi:10.1016/j.biopsych.2013.04.027.

31. Mathew SJ, Murrough JW, Aan het Rot M, Collins KA, Reich DL, Charney DS. Riluzole for relapse prevention following intravenous ketamine in treatment-resistant depression: a pilot randomized, placebo-controlled continuation trial. Int J Neuropsychopharmacol. 2010;13:71-82. doi:10.1017/S1461145709000169.
32. Ibrahim L, Diazgranados N, Franco-Chaves J, Brutsche N, Henter ID, Kronstein P, et al. Course of improvement in depressive symptoms to a single intravenous infusion of ketamine vs add-on riluzole: results from a 4-week, double-blind, placebo-controlled study. Neuropsychopharmacology. 2012;37(6):1526-33. doi:10. 1038/npp.2011.338.

33. Abdallah CG, Sanacora G, Duman RS, Krystal JH. Ketamine and rapid-acting antidepressants: a window into a new neurobiology for mood disorder therapeutics. Annu Rev Med. 2014;. doi:10. 1146/annurev-med-053013-062946.

34. Zarate CA Jr, Mathews DC, Furey ML. Human biomarkers of rapid antidepressant effects. Biol Psychiatry. 2013;73(12): 1142-55. doi:10.1016/j.biopsych.2012.11.031.

35. Heyes MP, Saito K, Crowley JS, Davis LE, Demitrack MA, Der $\mathrm{M}$, et al. Quinolinic acid and kynurenine pathway metabolism in inflammatory and non-inflammatory neurological disease. Brain. 1992;115(Pt 5):1249-73.

36. Erhardt S, Lim CK, Linderholm KR, Janelidze S, Lindqvist D, Samuelsson M, et al. Connecting inflammation with glutamate agonism in suicidality. Neuropsychopharmacology. 2012;. doi:10.1038/npp.2012.248.

37. Janelidze S, Mattei D, Westrin A, Traskman-Bendz L, Brundin L. Cytokine levels in the blood may distinguish suicide attempters from depressed patients. Brain Behav Immun. 2011;25(2):335-9. doi:10.1016/j.bbi.2010.10.010.

38. Mathew SJ, Charney DS. Publication bias and the efficacy of antidepressants. Am J Psychiatry. 2009;166:140.

39. Beck AT, Brown G, Berchick RJ, Stewart BL, Steer RA. Relationship between hopelessness and ultimate suicide: a replication with psychiatric outpatients. Am J Psychiatry. 1990;147(2): 190-5.

40. Luckenbaugh DA, Niciu MJ, Ionescu DF, Nolan NM, Richards EM, Brutsche NE, et al. Do the dissociative side effects of ketamine mediate its antidepressant effects? J Affect Disord. 2014;159:56-61. doi:10.1016/j.jad.2014.02.017.

41. Dakwar E, Anerella C, Hart CL, Levin FR, Mathew SJ, Nunes EV. Therapeutic infusions of ketamine: do the psychoactive effects matter? Drug Alcohol Depend. 2014;136:153-7. doi:10. 1016/j.drugalcdep.2013.12.019.

42. Schatzberg AF. A word to the wise about ketamine. Am J Psychiatry. 2014;171(3):262-4. doi:10.1176/appi.ajp.2014. 13101434.

43. Lally N, Nugent AC, Luckenbaugh DA, Ameli R, Roiser JP, Zarate CA. Anti-anhedonic effect of ketamine and its neural correlates in treatment-resistant bipolar depression. Transl Psychiatry. 2014;4:e469. doi:10.1038/tp.2014.105.

44. Nock MK, Park JM, Finn CT, Deliberto TL, Dour HJ, Banaji MR. Measuring the suicidal mind: implicit cognition predicts suicidal behavior. Psychol Sci. 2010;21:511-7. doi:10.1177/ 0956797610364762.

45. Harmer CJ, Goodwin GM, Cowen PJ. Why do antidepressants take so long to work? A cognitive neuropsychological model of antidepressant drug action. Br J Psychiatry. 2009;195:102-8. doi:10.1192/bjp.bp.108.051193. 